Artículo

\title{
Influencia de los valores humanos en el consumo de quesos tradicionales chiapanecos: una comparación de las rutas directa e indirecta
}

\author{
Carolina Illescas-Marin ${ }^{\text {a }}$ \\ Arturo Hernández-Montes ${ }^{a *}$ \\ Esaú Estrada-Estrada ${ }^{a}$ \\ Rolando Murguía-Cozar ${ }^{\text {a }}$ \\ Anastacio Espejel-García ${ }^{a}$ \\ Armando Santos-Moreno ${ }^{\text {a }}$
}

${ }^{a}$ Universidad Autónoma Chapingo. Departamento de Ingeniería Agroindustrial. Km 38.5

Carretera México-Texcoco. 56230, Chapingo, Estado de México, México.

* Autor de correspondencia: sensorial@ prodigy.net.mx

\section{Resumen:}

La preferencia de productos puede ser influenciada por valores humanos específicos y creencias individuales, entre otros factores. El propósito de la investigación fue identificar la influencia directa e indirecta de los valores humanos, mediados a través de los atributos tangibles, sobre la aceptación de los quesos Bola de Ocosingo y Crema de Chiapas. Se aplicaron encuestas a muestras $(n 1=200 ; n 2=230)$ de consumidores chiapanecos, para cada variedad de queso. Se obtuvieron coeficientes de correlación (R1) de regresiones de factores seleccionados de un análisis factorial de atributos tangibles y la frecuencia de consumo. Se calculó el R2, de las regresiones del consumo y los factores seleccionados de los valores humanos, junto con factores residuales de atributos tangibles. Los factores de solo los valores humanos y el consumo proporcionaron el R3. La influencia directa de los valores humanos en el consumo del queso se obtuvo restando R2 de R1 (cambio en R) y la influencia indirecta de la diferencia entre el coeficiente R3 y el cambio en R. La influencia directa no resultó significativa en el Queso Bola de Ocosingo, pero sí en el Queso Crema de Chiapas, por lo que los valores predijeron el consumo de este último 
queso más allá de la importancia de los atributos tangibles. Los valores motivacionales importantes en ambas muestras de consumidores fueron Benevolencia y Seguridad, adicionalmente, los consumidores del queso Crema de Chiapas presentaron el Poder y el Universalismo; mientras que los del queso Bola de Ocosingo mostraron al Hedonismo, como un valor suplementario.

Palabras clave: Chiapas, Valores humanos, Atributos intangibles.

Recibido: 06/03/2018

Aceptado: 29/08/2018

\section{Introducción}

Dentro de los quesos tradicionales mexicanos se encuentran dos quesos chiapanecos elaborados con leche cruda, el queso Crema de Chiapas y el queso Bola de Ocosingo ${ }^{(1,2)}$. Un alimento tradicional es "un producto frecuentemente consumido o asociado a una celebración o época, normalmente transmitida de una generación a otra, realizada con exactitud en una manera específica, de acuerdo a la herencia gastronómica, con o sin un procesamiento, distinguido y conocido debido a sus propiedades sensoriales y asociado con cierta área, región o país”(3). Los quesos artesanales son producidos de forma única, tradicional no industrial, a pequeña escala, con un grado limitado de mecanización ${ }^{(4)}$, en México se han descrito 31 quesos artesanales tradicionales ${ }^{(2)}$, estos están asociados a la crianza de animales, por parte de pequeños ganaderos, como una estrategia de ahorro y a la elaboración de quesos para asegurarle viabilidad al núcleo familiar ${ }^{(5,6)}$.

Los productos del mercado son una colección de atributos tangibles e intangibles; lo tangible se refiere a elementos del producto verificables objetivamente, y lo intangible es aquello que no altera la forma física del producto, pero ayuda a determinar su significado simbólico ${ }^{(7)}$. Thomson et al $^{(8)}$ señalaron que los consumidores, al procesar información sensorial obtienen conceptualizaciones funcionales, emocionales y abstractas; además, buscan construir símbolos, mostrando actitudes más favorables hacia productos cuando estos simbolizan los valores que ellos endorsan ${ }^{(9,10)}$. Los valores y las creencias son las unidades que construyen las actitudes en los consumidores ${ }^{(11)}$, los valores humanos son propósitos deseables que trascienden situaciones, los cuales varían en importancia, que sirven como principios guiadores en la vida de una persona $u$ otra entidad social ${ }^{(12,13)}$ y han sido empleados en el estudio de conductas de consumo de alimentos ${ }^{(14,15)}$ y en diferentes culturas, tales como la india ${ }^{(16)}$, la china ${ }^{(17)}$ y la europea ${ }^{(18)}$.

Para explicar la actitud hacia una conducta de consumo de un producto, el modelo de expectativa-valor se ha empleado en alimentos ${ }^{(19)}$ y en el consumo de carne ${ }^{(20,21)}$ 
empleando atributos tangibles como mediadores de la influencia de los valores humanos sobre la preferencia. Lindberg, et $a l^{(22)}$, encontraron que la preferencia de un producto era una función de las creencias de que el producto por completo debería reforzar a los valores humanos y su importancia. Keaveney y Hunt ${ }^{(23)}$ argumentaron que aunque la percepción de los atributos intangibles están embebidos en el conjunto de percepciones de los tangibles, la percepción de los atributos intangibles son más que la suma de las percepciones de los tangibles, y la distinción entre estos debería ser la consecuencia de la influencia de los valores humanos, mediando a través de ambas clases de atributos, para influir en la preferencia del producto. Cuando los consumidores evalúan el significado utilitario del producto, los valores humanos pueden influenciar la importancia de los atributos tangibles del producto, que a su vez influyen indirectamente en la preferencia del producto. Pero cuando los consumidores evalúan el significado simbólico a través de un juicio holístico afectivo, los valores humanos pueden influir en la preferencia del producto directamente, empleando a los atributos tangibles como mediadores. En la ruta indirecta, el producto sirve como una función psicológica instrumental y en la ruta directa el producto sirve como una función expresiva ${ }^{(24)}$. Por lo tanto, los consumidores nativos de queso deberán, para algunas variedades de quesos tradicionales, emplear más a los atributos intangibles que a los tangibles para expresar su conducta de consumo; pero para otras variedades de quesos, los consumidores podrán emplear solamente a los atributos tangibles en su decisión de consumo. El propósito de este trabajo fue identificar la ruta de influencia de los valores humanos en la frecuencia de consumo del queso Crema de Chiapas y el queso Bola de Ocosingo.

\section{Material y métodos}

Se empleó el procedimiento descrito por Schwartz ${ }^{(25)}$ y Allen ${ }^{(19)}$, el cual consistió en aplicar encuestas descriptivas de respuestas cerradas, empleando el programa Microsoft Access 2016 (Microsoft Corporation, USA). Para el caso de queso Crema de Chiapas se empleó una muestra de 230 consumidores nativos de los municipios de Pijijiapan, Tuxtla Gutiérrez y Comitán de Domínguez, Chiapas; y para el queso Bola de Ocosingo se empleó una muestra de 200 personas nativas del municipio de Ocosingo, Chiapas. En ambos casos los encuestados fueron consumidores mayores de 40 años y los géneros estuvieron balanceados. El muestreo fue de conveniencia para una población infinita y el tamaño de muestra se calculó empleando la máxima varianza, con una confiabilidad del $95 \%$ y un margen de error del $7 \%$. Las encuestas comprendieron tres secciones, en la primera sección, para el caso del queso Crema de Chiapas, se les presentó a cada persona una lista de 11 atributos tangibles (color amarillo, color blanco, firmeza, húmedo, desmoronabilidad, aroma lácteo, aroma a leche ácida, aroma a grasa, sabor ácido, sabor salado y precio accesible) y para el Queso Bola fueron precio accesible y 14 atributos tangibles, dos para la corteza (color amarillo y humedad), 12 para el relleno del queso (color crema, humedad, aroma lácteo, aroma a leche ácida, aroma a grasa, sabor ácido, 
sabor salado, sabor amargo, untabilidad, blandura, desmoronabilidad y granulosidad), los atributos se evaluaron empleando una escala de 10 puntos ( 1 "no es importante" y 10 "es muy importante"), de acuerdo a la importancia que tenía cada atributo para el encuestado, en la decisión de compra de cada queso.

La segunda sección de cada una de las encuestas consistió en identificar la importancia que los encuestados les otorgaban a los valores humanos. Para tal efecto, las personas leyeron previamente una hoja impresa con los 40 valores humanos y sus significados (Cuadro 1), en casos necesarios, el encuestador resolvió las dudas surgidas. Las personas inicialmente seleccionaron los 13 valores humanos más importantes; para luego seleccionar los 13 valores menos importantes. Se asignó un tres a los valores seleccionados como más importantes, un uno a los valores humanos menos importantes y finalmente un dos a aquellos valores no empleados por el encuestado. Esta metodología está basada en el estudio que realizó Schwartz ${ }^{(12)}$ con personas de 20 países $(n \geq 200$, por país) que incluyó todos los continentes, 13 idiomas, personas simpatizantes de ocho religiones, además de ateos; inicialmente trabajó con 11 valores motivacionales y 56 valores humanos, previamente consensuados por expertos en el tema; los resultados del estudio recomendaron el uso de 40 valores humanos asociados a diez valores motivacionales; además propuso su modelo de estructura de relaciones entre tipos de valores motivacionales, que tiene una amplia aplicación en estudios con valores humanos ${ }^{(14-18)}$. En la tercera sección de cada encuesta se les preguntó a los consumidores la frecuencia de consumo, en los últimos tres días, de cualquier tipo de los dos quesos investigados.

Cuadro 1: Valores motivacionales, valores humanos y su significado empleados en la encuesta a consumidores chiapanecos de quesos tradicionales

\begin{tabular}{lll}
\hline $\begin{array}{l}\text { Valores } \\
\text { motivacionales }\end{array}$ & Valores humanos & \multicolumn{1}{c}{ Definición } \\
\hline Auto-dirección & Auto determinación & Habilidad para determinar su propio destino \\
Auto-dirección & Auto respeto & Autoestima \\
Auto-dirección & Imaginativo & Atrevido, creativo \\
Auto-dirección & Independiente & Auto confiable, auto suficiente \\
Auto-dirección & Libertad & Independencia, selección libre \\
Benevolencia & Amistad verdadera & Compañerismo cercano \\
Benevolencia & Amor maduro & Intimidad espiritual y sexual \\
Benevolencia & Cariñoso & Afectivo, tierno \\
Benevolencia & Honesto & Sincero, creíble \\
Benevolencia & Indulgencia & Deseo de perdonar a otros \\
Benevolencia & Responsable & Digno de confianza, confiable \\
Benevolencia & Salvación & Salvado, vida eterna \\
Benevolencia & Útil & Trabajar para el bienestar de otros \\
Conformidad & Auto controlado & Refrenado, auto disciplinado
\end{tabular}




\begin{tabular}{lll} 
Conformidad & Educado & Cortes, buena manera \\
Universalismo & Equidad & $\begin{array}{l}\text { Cada persona es gratificada de acuerdo a cuanto } \\
\text { haya realizado }\end{array}$ \\
Conformidad & Obediente & Con obligaciones, respetable \\
Estimulación & Una vida excitante & Una vida estimulante, vida activa \\
Estimulación & Valiente & De pie por tus convicciones \\
Hedonismo & Alegre & Poco serio \\
Hedonismo & Felicidad & Sentirse contento \\
Hedonismo & Placentero & Agradable, vida relajada \\
Hedonismo & Una vida confortable & Una vida próspera \\
Logro & Ambicioso & Trabajar duro, aspirar \\
Logro & Capaz & Competente, efectivo \\
Logro & Intelectual & Inteligente, reflexivo \\
Logro & Lógico & Consistente, racional \\
Logro & Un sentido de logro & Contribución duradera \\
Poder & Poder social & Posición de autoridad e importancia \\
Poder & Reconocimiento social & Respeto, admiración \\
Seguridad & Limpio & Ordenado, pulcro \\
Seguridad & Seguridad familiar & Tomar cuidado de los seres queridos \\
Seguridad & Seguridad nacional & Protección de ataque \\
Tradición & Respeto por la tradición & Compromiso y aceptación de las costumbres \\
Universalismo & Armonía interior & Libre de conflictos internos \\
Universalismo & Igualdad & Comunidad, oportunidad igual para todos \\
Universalismo & Justicia social & Rectitud, no discriminación \\
Universalismo & Mente amplia & Mente abierta \\
Universalismo & Sabiduría & Un entendimiento maduro de la vida \\
Universalismo & Un mundo de paz & Libre de guerras y conflictos \\
\hline & & \\
\hline
\end{tabular}

Fuente: Schwartz ${ }^{(12)}$

\section{Análisis estadístico}

El procedimiento estadístico se realizó para cada una de las dos variedades de queso, un primer paso fue aplicar un análisis factorial sin rotación, empleando el método de componentes principales, para los valores humanos y otro para los atributos tangibles del queso investigado, con la finalidad de reducir el número de variables (40 valores humanos y atributos tangibles de los quesos); el criterio para seleccionar a los factores fue que los eigenvalores fueran iguales o mayores a uno. Posteriormente, se realizó una regresión múltiple (bloque uno) con los factores seleccionados de los atributos tangibles (variables independientes) y la frecuencia de consumo de queso (variable dependiente). Una segunda regresión (bloque dos) se aplicó a la frecuencia de consumo de queso y a los factores seleccionados de los valores humanos, junto con los factores residuales de los atributos tangibles (eigenvalores menores de uno), los cuales fueron las variables 
independientes. Una tercera y última regresión múltiple se realizó con los factores seleccionados de los valores humanos y la frecuencia del consumo del queso, a esto se le llamó la regresión de solo los valores humanos. Las regresiones múltiples se llevaron a cabo empleando el método por pasos con un $\alpha=0.1$, tanto para incluir como para excluir a las variables independientes, con el programa XLSTAT versión 2014 (Addinsoft, USA). De cada una de las tres regresiones, para cada queso, se obtuvieron los análisis de varianza y los coeficientes de regresión múltiple $(\mathrm{R})$.

Para obtener la influencia directa de los valores humanos en la frecuencia de consumo del queso, con los atributos tangibles como mediadores, el $\mathrm{R}$ de la regresión del bloque uno se substrajo del $\mathrm{R}$ de la regresión del bloque dos (cambio en $\mathrm{R}$ ). Para evaluar la significancia del cambio en $\mathrm{R}$, los coeficientes de regresión se transformaron a $\mathrm{z}$ ' de Fisher ${ }^{(26)}$, para luego aplicar una prueba de $\mathrm{z}$ para evaluar la hipótesis nula de que un $\mathrm{R}$ es igual a un valor específico $\left(\varphi_{1}=\varphi_{\mathrm{k}}\right)$, empleando el programa SAS versión 9.4 (SAS Institute, Inc., Cary, NC). La influencia indirecta de los valores humanos sobre la frecuencia de consumo (empleando a los atributos tangibles como mediadores) de cada uno de los quesos se obtuvo de la diferencia del coeficiente de regresión de solo los valores humanos y el cambio en $\mathrm{R}^{(19,24)}$.

\section{Resultados}

\section{Análisis factoriales de los atributos tangibles de los quesos y de valores humanos en consumidores chipanecos}

El análisis factorial para los atributos tangibles del queso crema de Chiapas proporcionó tres factores con eigenvalores mayores o iguales a uno, los cuales explicaron el $49.86 \%$ de la variación total de los datos. Para el queso Bola de Ocosingo resultó con cinco factores (eigenvalores $\geq 1$ ) que explicaron el $62.04 \%$ de la variación total de la información.

El análisis factorial de los valores humanos en los consumidores del queso Crema de Chiapas mostró 14 factores que explicaron el $60.4 \%$ de la variabilidad total y para el Queso Bola de Ocosingo, 15 factores resultaron con valores propios iguales o mayores a uno y explicaron el $64.2 \%$ de la variación total de la información de los valores humanos. 


\section{Influencia directa e indirecta de los valores humanos en los consumos del queso Crema de Chiapas y Bola de Ocosingo}

A las coordenadas de las observaciones de cada uno de los factores seleccionados de los atributos tangibles para cada variedad de queso y de cada uno de los factores seleccionados de los valores humanos de las dos muestras de consumidores chiapanecos se les aplicó la prueba de normalidad de Kolmogorov-Smirnov ${ }^{(27)}$ y para todos los casos no se rechazó la hipótesis del supuesto de distribución normal $(P>0.05)$. Los resultados de las tres regresiones múltiples relacionadas con el queso Crema de Chiapas se muestran en el Cuadro 2; la regresión de los tres factores que contenían atributos tangibles y la frecuencia de consumo suministró como variable significativa $(P<0.1)$ al factor dos $(\mathrm{T} 2)$, la regresión de los 14 factores de valores humanos más los residuales de los factores intangibles (RT4 - RT11) y la frecuencia de consumo como variable dependiente proporcionó cuatro variables significativas $(P<0.1)$, tres factores de valores humanos (V3, V4 y V5) y un factor residual de atributos tangibles (RT8). De la regresión múltiple del bloque tres, la cual incluyó los 14 factores de valores humanos y el consumo de queso, se obtuvieron tres variables o factores (V3, V4 y V5). Las regresiones múltiples relacionadas con el queso Bola de Ocosingo se muestran en el Cuadro 3, la regresión de los cinco factores que contenían atributos tangibles y la frecuencia de consumo suministró tres variables significativas $(P<0.1)$ que resultaron ser los factores T1, T2 y T3, la regresión de los 15 factores de valores humanos más los residuales de los factores intangibles (RT6 - RT13) y la frecuencia de consumo como variable dependiente proveyó tres variables significativas $(P<0.1)$, dos factores de valores humanos (V7 y V13) y un factor residual de tangibles (RT8); la regresión múltiple que incluyó los 15 factores conteniendo valores humanos y el consumo de queso como variable dependiente seleccionó dos variables o factores (V7 y V13) significativas en el modelo.

Cuadro 2: Regresiones múltiples realizadas con los datos de los valores humanos de consumidores chiapanecos y los atributos tangibles del queso Crema de Chiapas

\begin{tabular}{lccccc}
\hline & Fuente & $\begin{array}{c}\text { Valor } \\
\text { Coeficiente (B) }\end{array}$ & $\begin{array}{c}\text { Error } \\
\text { estándar }\end{array}$ & $\mathbf{t}$ & $\operatorname{Pr}>|\mathbf{t}|$ \\
\hline Regresión de los factores de & Intersección & 3.530 & 0.111 & 31.708 & $<0.0001$ \\
atributos tangibles y el & T1 & 0.000 & 0.000 & & \\
consumo de queso & T2 & 0.268 & 0.084 & 3.200 & 0.002 \\
& T3 & 0.000 & 0.000 & &
\end{tabular}

Ecuación de regresión (R1)

Consumo $=3.530+0.268^{\star} \mathrm{T} 2$

$\begin{array}{lccccc} & \text { Intersección } & 3.530 & 0.109 & 32.428 & <0.0001 \\ \text { Regresión de los factores de } & \text { RT4 } & 0.000 & 0.000 & & \\ \text { los residuales de los atributos } & \text { RT5 } & 0.000 & 0.000 & \end{array}$


Rev Mex Cienc Pecu 2019;10(4):966-985

tangibles más los factores de los valores humanos y el consumo de queso

Regresión de los factores de los valores humanos y el consumo

Ecuación de regresión (R2)
RT6

RT7

RT8

RT9

RT10

RT11

V1

V2

V3

V4

V5

V6

V7

V8

V9

V10

V11

V12

V13

V14

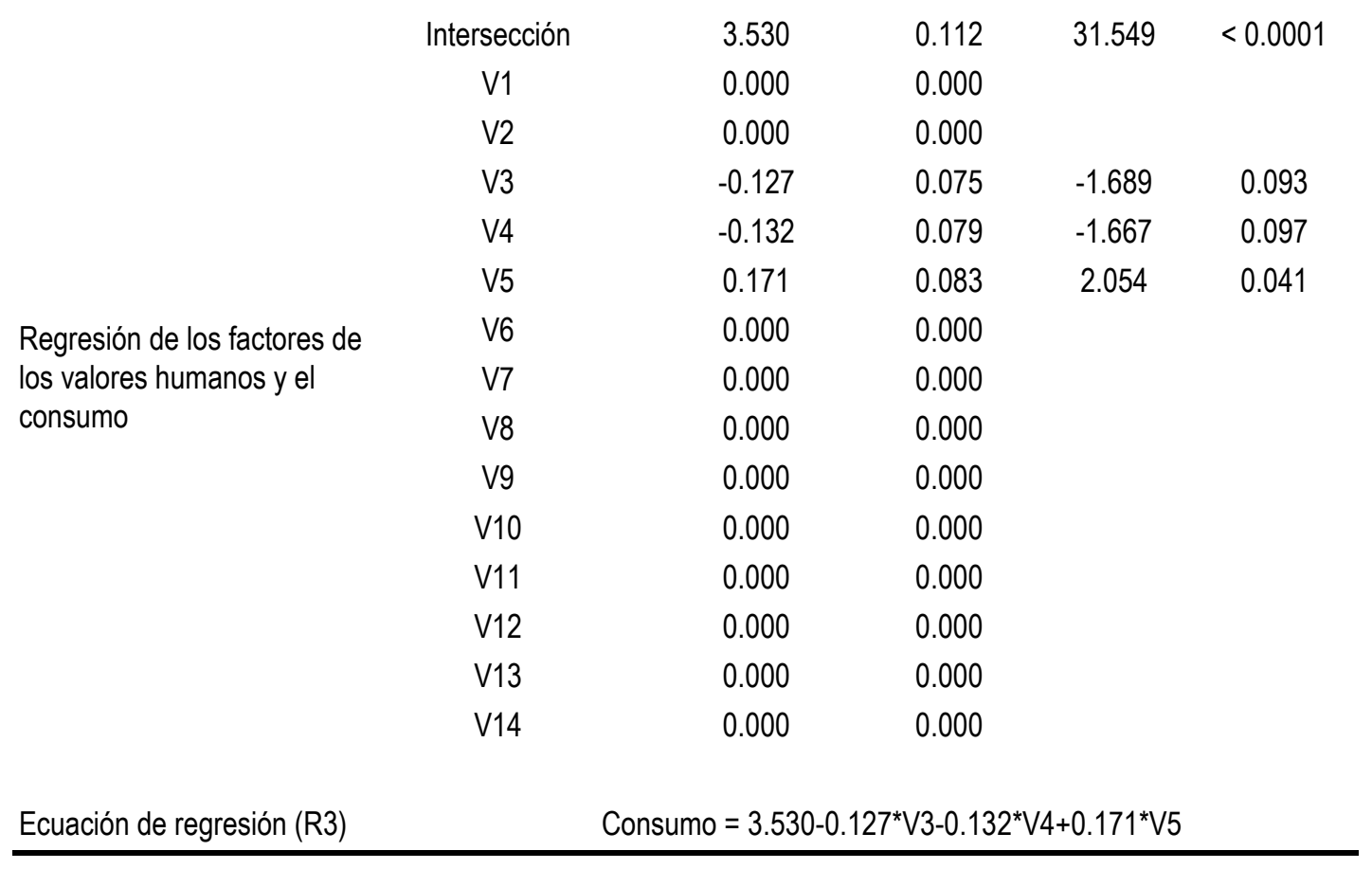


Cuadro 3: Regresiones múltiples realizadas con los datos de los valores humanos de consumidores chiapanecos y los atributos tangibles del queso Bola de Ocosingo

\begin{tabular}{|c|c|c|c|c|c|}
\hline & Fuente & $\begin{array}{c}\text { Valor } \\
\text { Coeficiente (B) }\end{array}$ & $\begin{array}{c}\text { Error } \\
\text { estándar }\end{array}$ & $t$ & $\operatorname{Pr}>|t|$ \\
\hline \multirow{4}{*}{$\begin{array}{l}\text { Regresión de los factores de } \\
\text { atributos tangibles y el consumo de } \\
\text { queso }\end{array}$} & Intersección & 3.1100 & 0.1262 & 24.6481 & $<0.0001$ \\
\hline & $\mathrm{T} 1$ & 0.0000 & 0.0000 & & \\
\hline & $\mathrm{T} 2$ & 0.0000 & 0.0000 & & \\
\hline & T3 & -0.3282 & 0.0988 & -3.3216 & 0.0011 \\
\hline \multirow[t]{12}{*}{ Ecuación de regresión (R1) } & \multicolumn{5}{|c|}{ Consumo $=3.110-0.328 * \mathrm{~T} 3$} \\
\hline & Intersección & 3.1100 & 0.1251 & 24.8671 & $<0.0001$ \\
\hline & V1 & 0.0000 & 0.0000 & & \\
\hline & V2 & 0.0000 & 0.0000 & & \\
\hline & V3 & 0.0000 & 0.0000 & & \\
\hline & \multicolumn{5}{|c|}{ V4 } \\
\hline & V5 & 0.0000 & 0.0000 & & \\
\hline & V6 & 0.0000 & 0.0000 & & \\
\hline & V7 & 0.2024 & 0.0995 & 2.0334 & 0.0434 \\
\hline & V8 & 0.0000 & 0.0000 & & \\
\hline & V9 & 0.0000 & 0.0000 & & \\
\hline & V10 & 0.0000 & 0.0000 & & \\
\hline \multirow{13}{*}{$\begin{array}{l}\text { Regresión de los factores de los } \\
\text { residuales de los atributos tangibles } \\
\text { más los factores de los valores } \\
\text { humanos y el consumo de queso }\end{array}$} & V11 & 0.0000 & 0.0000 & & \\
\hline & V12 & 0.0000 & 0.0000 & & \\
\hline & V13 & 0.3544 & 0.1182 & 2.9989 & 0.0031 \\
\hline & V14 & 0.0000 & 0.0000 & & \\
\hline & V15 & 0.0000 & 0.0000 & & \\
\hline & RT6 & 0.0000 & 0.0000 & & \\
\hline & RT7 & 0.0000 & 0.0000 & & \\
\hline & RT8 & -0.3209 & 0.1551 & -2.0696 & 0.0398 \\
\hline & RT9 & 0.0000 & 0.0000 & & \\
\hline & RT10 & 0.0000 & 0.0000 & & \\
\hline & RT11 & 0.0000 & 0.0000 & & \\
\hline & RT12 & 0.0000 & 0.0000 & & \\
\hline & RT13 & 0.0000 & 0.0000 & & \\
\hline
\end{tabular}

Intersección

3.1100

0.1261

$24.6625<0.0001$

$\begin{array}{lll}\mathrm{V} 1 & 0.0000 \quad 0.0000\end{array}$




\begin{tabular}{ccccc}
\hline V2 & 0.0000 & 0.0000 & & \\
V3 & 0.0000 & 0.0000 & & \\
V4 & 0.0000 & 0.0000 & & \\
V5 & 0.0000 & 0.0000 & & \\
V6 & 0.0000 & 0.0000 & 0.0636 \\
V7 & 0.1867 & 0.1001 & 1.8656 & \\
V8 & 0.0000 & 0.0000 & & \\
V9 & 0.0000 & 0.0000 & & \\
V10 & 0.0000 & 0.0000 & & \\
V11 & 0.0000 & 0.0000 & & \\
V12 & 0.0000 & 0.0000 & & \\
V13 & 0.3534 & 0.1191 & 2.9660 & 0.0034 \\
V14 & 0.0000 & 0.0000 & & \\
V15 & 0.0000 & 0.0000 & & \\
\hline
\end{tabular}

Los análisis de varianza de las tres regresiones relacionadas con cada uno de las dos variedades de quesos indicaron que los modelos fueron significativos y los coeficientes de correlación se muestran en los Cuadros 4 y 5; para el queso Crema de Chiapas la diferencia (cambio en $\mathrm{R}$ ) entre los coeficientes de correlación de la regresión de bloque dos y el coeficiente del bloque uno fue de 0.104 y resultó significativo $(P<0.05)$. Esto indica el grado en el que los valores humanos predijeron la aceptación del queso Crema de Chiapas, más allá de las importancias de los atributos tangibles, por lo tanto, representa la influencia vía ruta directa de los valores humanos que fueron expresados a través de la importancia de los atributos intangibles. Para el queso Bola de Ocosingo, la diferencia (cambio en R) entre los coeficientes de correlación de la regresión del bloque dos y del bloque uno fue de 0.051 y no resultó significativa $(P>0.05)$, esto indica el grado en que los valores humanos (ruta directa) no predijeron la aceptación del queso Bola de Ocosingo, más allá de las importancias de los atributos tangibles. 
Cuadro 4: Resultados de las regresiones de la importancia de los atributos tangibles, y de los valores humanos y residuales de atributos tangibles en el consumo de dos quesos tradicionales chiapanecos

\begin{tabular}{|c|c|c|c|c|c|c|c|}
\hline \multirow{3}{*}{$\begin{array}{l}\text { Variedad } \\
\text { de queso }\end{array}$} & \multirow{2}{*}{\multicolumn{3}{|c|}{$\begin{array}{c}\text { Bloque } 1 \\
\text { Atributos tangibles }\end{array}$}} & \multicolumn{3}{|c|}{ Bloque 2} & \multirow{3}{*}{$\begin{array}{l}\text { Cambio } \\
\text { en } R\end{array}$} \\
\hline & & & & \multicolumn{3}{|c|}{$\begin{array}{c}\text { Valores humanos más residuales de } \\
\text { atributos tangibles }\end{array}$} & \\
\hline & $\begin{array}{l}\text { Factores } \\
\text { Introducidos a }\end{array}$ & $\begin{array}{l}\text { Coeficientes } \\
\quad(\beta)\end{array}$ & R múltiple & $\begin{array}{l}\text { Factores } \\
\text { Introducidos a }\end{array}$ & $\begin{array}{l}\text { Coeficientes } \\
\quad(\beta)\end{array}$ & R múltiple & \\
\hline \multirow[t]{2}{*}{$\begin{array}{l}\text { Queso } \\
\text { Crema de } \\
\text { Chiapas }\end{array}$} & $\begin{array}{c}\mathrm{T} 2 \\
\text { (color amarillo, } \\
\text { aroma a leche y } \\
\text { aroma a grasa) }\end{array}$ & 0.268 & $\begin{array}{c}0.207 \\
F=10.24 \\
\text { g.l. }=1,228\end{array}$ & $\begin{array}{c}\text { RT8 } \\
\text { (salado) }^{\mathrm{b}}\end{array}$ & -0.505 & $\begin{array}{c}0.311 \\
F=6.04 \\
\text { g.l. }=4,225\end{array}$ & $\begin{array}{c}0.104 \\
z=1.68 \\
p= \\
0.046\end{array}$ \\
\hline & & & $(p=0.002)$ & 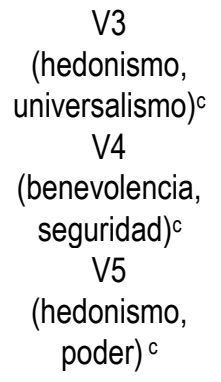 & $\begin{array}{l}-0.124 \\
-0.135\end{array}$ & $(p=0.002)$ & \\
\hline \multirow[t]{2}{*}{$\begin{array}{l}\text { Queso } \\
\text { Bola de } \\
\text { Ocosingo }\end{array}$} & $\begin{array}{c}\text { T3 } \\
\text { (precio } \\
\text { accesible) }\end{array}$ & -0.229 & $\begin{array}{c}0.229 \\
F=11.03 \\
\text { g.l. }=1,198\end{array}$ & $\begin{array}{c}\text { RT8 } \\
\text { (blandura) }^{c}\end{array}$ & -0.142 & $\begin{array}{c}0.280 \\
F=5.58 \\
g . l=3,196\end{array}$ & $\begin{array}{c}0.051 \\
z=0.76 \\
p= \\
0.223\end{array}$ \\
\hline & & & $(p=0.001)$ & $\begin{array}{c}\text { V7 } \\
\text { (benevolencia)c } \\
\text { V13 } \\
\text { (benevolencia)c }\end{array}$ & $\begin{array}{l}0.139 \\
0.205\end{array}$ & $(p=0.001)$ & \\
\hline
\end{tabular}

${ }^{\mathrm{a}}=$ solo se incluyeron factores significativos a una $\mathrm{p} \leq 0.1$

$\mathrm{b}=$ Atributos tangibles.

$\mathrm{c}=$ Valores motivacionales

Bloque 1 = importancia de atributos tangibles sobre la aceptabilidad del producto.

Bloque 2 = valores humanos sobre los remanentes de la aceptabilidad del producto, no tomados en cuenta por la importancia de los atributos tangibles. 
Cuadro 5: Resultados de la regresión de únicamente los valores humanos en el consumo de dos quesos chiapanecos

\begin{tabular}{|c|c|c|c|c|}
\hline \multirow{2}{*}{$\begin{array}{l}\text { Variedad de } \\
\text { queso }\end{array}$} & \multicolumn{2}{|c|}{ Valores humanos } & \multirow[b]{2}{*}{ R múltiple } & \multirow[b]{2}{*}{$\begin{array}{c}\text { Ruta } \\
\text { indirecta }\end{array}$} \\
\hline & Factores introducidosa & $\begin{array}{c}\text { Coeficientes } \\
(\beta)\end{array}$ & & \\
\hline $\begin{array}{l}\text { Queso Crema de } \\
\text { Chiapas }\end{array}$ & $\begin{array}{c}\text { V3 } \\
\text { (hedonismo, universalismo) }^{\mathrm{b}}\end{array}$ & -0.127 & $\begin{array}{c}0.204 \\
F=3.28 \text { g.l. }=3,226 \\
(p=0.022)\end{array}$ & 0.100 \\
\hline & $\begin{array}{c}\text { V4 } \\
\text { (benevolencia, seguridad) } \\
\text { V5 } \\
{\text { (hedonismo, poder })^{b}}^{b}\end{array}$ & $\begin{array}{l}-0.132 \\
0.171\end{array}$ & & \\
\hline
\end{tabular}

\begin{tabular}{|c|c|c|c|c|}
\hline \multirow[t]{2}{*}{$\begin{array}{l}\text { Queso Bola de } \\
\text { Ocosingo }\end{array}$} & $\begin{array}{c}\text { V7 } \\
\text { (benevolencia) }\end{array}$ & 0.186 & $\begin{array}{c}0.242 \\
F=6.13 \\
\text { g.l. }=2,197(0.002)\end{array}$ & 0.191 \\
\hline & $\begin{array}{c}\text { V13 } \\
\text { (benevolencia) }\end{array}$ & 0.353 & & \\
\hline
\end{tabular}

Por otro lado, los análisis de varianza de solo los valores humanos y el consumo tanto del queso Crema de Chiapas, como del queso Bola de Ocosingo (Cuadro 5) fueron significativos $(P<0.05)$. Para el queso Crema de Chiapas, el coeficiente de correlación de la regresión de solo los valores humanos (0.204), menos el cambio en R (0.104) resultó en 0.10 , lo cual representa la influencia de los valores humanos, por la vía de las importancias de los atributos tangibles (ruta indirecta), en la preferencia del queso. El coeficiente de correlación de la regresión de solo los valores humanos y la frecuencia de consumo del queso Bola de Ocosingo fue de 0.242 , por lo que al substraerle el cambio en $\mathrm{R}(0.051)$ resultó una influencia indirecta de 0.191 .

En la regresión del bloque uno, para el queso Crema de Chiapas, los atributos tangibles (Cuadro 6) con las mayores correlaciones positivas con la variable (factor T2) fueron color amarillo, aroma a leche ácida y aroma a grasa y con la mayor correlación negativa fue el color blanco. En la regresión del bloque dos, la variable residual ocho de los atributos tangibles (RT8) se correlacionó positivamente con el sabor ácido, el precio accesible y la humedad, pero negativamente con el sabor salado; y los valores motivacionales (Cuadro 7) con mayor correlación con la variable tres pertenecieron a los del Poder (poder social) y al Universalismo (justicia social); y con las mayores correlaciones negativas fueron el Hedonismo (alegre) y el Universalismo (mente amplia). La variable cuatro se correlacionó positivamente con los valores motivacionales de la Benevolencia (útil) y la Seguridad (limpio); y negativamente con el valor motivacional del Logro (intelectual). La variable cinco se correlacionó positivamente con los valores motivacionales de Hedonismo (placentero) y de Poder (poder social). 
Cuadro 6: Correlaciones de los atributos tangibles del "queso crema de Chiapas" con las variables (factores) seleccionadas en el análisis de regresión múltiple

\begin{tabular}{lcc}
\hline & T2 & RT8 \\
\hline Color amarillo & 0.815 & -0.049 \\
Color blanco & -0.658 & -0.026 \\
Firmeza & 0.109 & -0.202 \\
Desmoronable & -0.005 & -0.171 \\
Húmedo & -0.167 & 0.241 \\
Aroma lácteo & 0.119 & 0.108 \\
Aroma a leche ácida & 0.547 & 0.062 \\
Aroma a grasa & 0.485 & 0.092 \\
Sabor ácido & 0.120 & 0.296 \\
Sabor salado & -0.041 & -0.572 \\
Precio accesible & -0.248 & 0.266 \\
\hline
\end{tabular}

Cuadro 7: Correlaciones de los valores humanos en consumidores de queso Crema de Chiapas, con las variables (factores) seleccionadas en el análisis de regresión múltiple

\begin{tabular}{lllll}
\hline Valor motivacional & Valor humano & $\mathbf{V 3}$ & $\mathbf{V 4}$ & V5 \\
\hline Estimulación & Valiente & 0.280 & 0.245 & -0.084 \\
Benevolencia & Útil & 0.125 & 0.403 & -0.051 \\
Estimulación & Una vida excitante & 0.207 & 0.064 & -0.074 \\
Hedonismo & Una vida confortable & 0.181 & 0.248 & -0.230 \\
Logro & Un sentido de logro & 0.168 & -0.078 & 0.018 \\
Universalismo & Un mundo de paz & 0.173 & 0.276 & -0.149 \\
Conformidad & Auto controlado & -0.046 & -0.251 & -0.083 \\
Seguridad & Seguridad Nacional & -0.047 & -0.242 & -0.149 \\
Seguridad & Seguridad familiar & -0.063 & 0.250 & -0.340 \\
Benevolencia & Salvación & -0.270 & 0.200 & 0.041 \\
Universalismo & Sabiduría & -0.385 & -0.130 & 0.032 \\
Benevolencia & Responsable & -0.056 & 0.206 & 0.012 \\
Poder & Reconocimiento social & 0.304 & -0.088 & 0.398 \\
Poder & Poder social & 0.358 & -0.270 & 0.423 \\
Hedonismo & Placentero & -0.047 & -0.053 & 0.437 \\
Conformidad & Obediente & 0.099 & 0.322 & 0.347 \\
Universalismo & Mente amplia & -0.474 & -0.187 & 0.256 \\
Logro & Lógico & -0.205 & -0.305 & -0.213 \\
Seguridad & Limpio & -0.372 & 0.384 & -0.025 \\
Auto-dirección & Libertad & -0.325 & -0.257 & 0.103 \\
Universalismo & Justicia social & 0.384 & -0.183 & -0.021
\end{tabular}




\begin{tabular}{lllll} 
Logro & Intelectual & -0.035 & -0.386 & -0.243 \\
Benevolencia & Indulgencia & 0.125 & -0.167 & 0.289 \\
Auto-dirección & Independiente & -0.027 & -0.296 & 0.272 \\
Auto-dirección & Imaginativo & -0.261 & 0.143 & -0.353 \\
Universalismo & Igualdad & 0.373 & -0.087 & -0.022 \\
Benevolencia & Honesto & -0.028 & 0.283 & 0.146 \\
Hedonismo & Felicidad & 0.046 & 0.329 & 0.136 \\
Universalismo & Equidad & 0.257 & -0.271 & -0.228 \\
Conformidad & Educado & 0.023 & 0.234 & 0.046 \\
Benevolencia & Cariñoso & -0.140 & 0.138 & 0.156 \\
Logro & Capaz & 0.188 & -0.041 & -0.159 \\
Auto-dirección & Auto respeto & 0.034 & -0.035 & -0.093 \\
Auto-dirección & Auto determinación & 0.023 & -0.287 & -0.193 \\
Tradición & Respeto por la tradición & -0.209 & 0.077 & 0.155 \\
Universalismo & Armonía interior & 0.142 & -0.064 & 0.084 \\
Benevolencia & Amor maduro & -0.360 & -0.170 & -0.211 \\
Benevolencia & Amistad verdadera & 0.142 & 0.160 & -0.029 \\
Logro & Ambicioso & 0.238 & -0.098 & -0.418 \\
Hedonismo & Alegre & -0.497 & 0.021 & 0.067 \\
\hline
\end{tabular}

Para el queso Bola de Ocosingo los atributos tangibles, en la regresión del bloque uno (Cuadro 8), con las mayores correlaciones positivas con la variable tres (factor T3) fueron precio accesible y blandura; y con la mayor correlación negativa fue sabor amargo. En la regresión del bloque dos, la variable ocho de los atributos tangibles residuales (RT8) se correlacionó positivamente con el sabor ácido y negativamente con la blandura (Cuadro 8); y los valores motivacionales con mayor correlación con la variable siete (Cuadro 9) pertenecieron a los de Benevolencia (responsable y honesto) y Seguridad (seguridad nacional); y con las mayores correlaciones negativas fueron Universalismo (sabiduría) y Auto-dirección (imaginativo). La variable trece se correlacionó positivamente con el valor motivacional del Hedonismo (alegre) y negativamente con el Logro (intelectual) y el Poder (reconocimiento social). 
Cuadro 8: Correlaciones de los atributos tangibles del queso Bola de Ocosingo, con las variables (factores) seleccionadas en los análisis de regresión múltiple

\begin{tabular}{lcr}
\hline & T3 & RT8 \\
\hline Color amarillo en corteza & 0.0838 & -0.0310 \\
Humedad corteza & 0.0180 & -0.0315 \\
Color crema & 0.2442 & -0.1415 \\
Humedad & 0.1246 & 0.1670 \\
Aroma lácteo & -0.2932 & 0.2590 \\
Aroma a leche ácida & -0.2383 & -0.0386 \\
Aroma a grasa & -0.4330 & -0.2053 \\
Sabor ácido & 0.2464 & 0.4768 \\
Sabor salado & 0.0405 & 0.1229 \\
Sabor amargo & -0.6424 & -0.0203 \\
Untabilidad & 0.2650 & -0.0957 \\
Blandura & 0.4221 & -0.4709 \\
Granulosidad & -0.2349 & 0.0111 \\
Desmoronabilidad & -0.3181 & -0.1116 \\
Precio accesible & 0.5815 & 0.0868 \\
\hline
\end{tabular}

Cuadro 9: Correlaciones de los valores humanos en consumidores de queso Bola de Ocosingo, con las variables (factores) seleccionadas en el análisis de regresión múltiple

\begin{tabular}{llcc}
\hline Valores motivacionales & Valores humanos & V7 & V13 \\
\hline Estimulación & Valiente & 0.0117 & -0.0244 \\
Benevolencia & Útil & -0.2558 & 0.3224 \\
Estimulación & Una vida excitante & 0.1042 & 0.2111 \\
Hedonismo & Una vida confortable & -0.2390 & 0.1227 \\
Logro & Un sentido de logro & 0.0953 & 0.1028 \\
Universalismo & Un mundo de paz & -0.0084 & -0.0828 \\
Conformidad & Auto controlado & -0.2979 & -0.2645 \\
Seguridad & Seguridad Nacional & $\mathbf{0 . 3 7 3 9}$ & 0.1018 \\
Seguridad & Seguridad familiar & -0.0940 & 0.1015 \\
Benevolencia & Salvación & 0.2474 & -0.0757 \\
Universalismo & Sabiduría & $\mathbf{- 0 . 4 3 1 1}$ & 0.0762 \\
Benevolencia & Responsable & $\mathbf{0 . 3 6 2 7}$ & 0.0568 \\
Poder & Reconocimiento social & 0.0245 & $\mathbf{- 0 . 3 0 1 9}$ \\
Poder & Poder social & 0.0602 & 0.0260 \\
Hedonismo & Placentero & 0.0289 & -0.0965 \\
Conformidad & Obediente & 0.0532 & -0.0380 \\
Universalismo & Mente amplia & -0.0529 & 0.0797 \\
Logro & Lógico & -0.0754 & -0.2071
\end{tabular}




\begin{tabular}{llcc}
\hline & & & \\
Seguridad & Limpio & 0.0109 & -0.0740 \\
Auto-dirección & Libertad & 0.0721 & -0.0385 \\
Universalismo & Justicia social & 0.0882 & -0.1271 \\
Logro & Intelectual & -0.1513 & $\mathbf{- 0 . 4 1 6 0}$ \\
Benevolencia & Indulgencia & 0.2883 & -0.0279 \\
Auto-dirección & Independiente & 0.2518 & 0.1944 \\
Auto-dirección & Imaginativo & $\mathbf{- 0 . 3 2 5 4}$ & 0.0974 \\
Universalismo & Igualdad & 0.0050 & -0.0330 \\
Benevolencia & Honesto & $\mathbf{0 . 3 1 9 1}$ & -0.3177 \\
Hedonismo & Felicidad & 0.1565 & 0.0689 \\
Universalismo & Equidad & -0.0023 & -0.1587 \\
Conformidad & Educado & 0.1183 & -0.0745 \\
Benevolencia & Cariñoso & 0.2397 & 0.0480 \\
Logro & Capaz & 0.0149 & 0.1555 \\
Auto-dirección & Auto respeto & -0.2436 & 0.1822 \\
Auto-dirección & Auto determinación & -0.1547 & -0.1493 \\
Tradición & Respeto por la tradición & -0.0396 & 0.2495 \\
Universalismo & Armonía interior & 0.1170 & 0.0689 \\
Benevolencia & Amor maduro & -0.2948 & 0.0550 \\
Benevolencia & Amistad verdadera & 0.1880 & 0.0277 \\
Logro & Ambicioso & -0.1301 & 0.2475 \\
Hedonismo & Alegre & -0.2977 & $\mathbf{0 . 5 4 1 4}$ \\
\hline & & &
\end{tabular}

\section{Discusión}

El queso Crema de Chiapas, a diferencia del queso de Bola de Ocosingo mostró una influencia directa de los valores humanos en su consumo, lo cual indica que los consumidores del queso Crema de Chiapas emplearon atributos intangibles, además de los tangibles, para expresar la influencia de los valores en el consumo del queso, empleando a los atributos tangibles como mediadores; los valores motivacionales mayormente involucrados fueron los del Poder, el Universalismo, la Benevolencia, el Hedonismo, y la Seguridad; los valores de Universalismo y Benevolencia son valores motivacionales adyacentes a los de la Tradición y la Conformidad, sin embargo el Hedonismo y el Poder en menor medida son opuestos a los valores de la Tradición y la Conformidad, los cuales serían esperados en una comunidad tradicionalista ${ }^{(12)}$. Este resultado coincide con lo publicado para carnes rojas, debido a que los consumidores emplearon atributos intangibles (simbólicos y afectivos) en su selección ${ }^{(21)}$, en Brasil se reportó que la carne roja simbolizaba jerarquía social y que su consumo estaba relacionado con los valores del Poder ${ }^{(20)}$; en Australia se encontró que el consumo de carne estaba relacionado con los valores asociados al Universalismo, el Poder y la Seguridad; las personas que presentaban una actitud positiva al consumo de carne roja 
expresaron como más importantes los valores relacionados con el Poder y la Seguridad $^{(21)}$. En un estudio de bebidas saludables realizado en China los valores motivacionales encontrados en una muestra de consumidores fueron los de Seguridad, Hedonismo, Benevolencia y Logro ${ }^{(17)}$. Las comparaciones de las estructuras de los valores humanos y motivacionales, entre consumidores de diferentes culturas son posibles, por ejemplo, los consumidores que proporcionan prioridad a los valores motivacionales de la Conservación y Tradición tendrán como valores opuestos, a los valores motivacionales del Logro y el Hedonismo; los consumidores que prefieren la carne roja priorizan los valores motivacionales del Poder y el Logro, sobre los de la Benevolencia y el Universalismo ${ }^{(16,17,20,21)}$.

Por otro lado, la influencia de los valores humanos de los consumidores del queso Bola de Ocosingo empleó totalmente a los atributos tangibles para mostrar su preferencia. Los principales valores motivacionales incluidos en la ruta indirecta del queso Bola de Ocosingo incluyeron a los de la Benevolencia, la Seguridad y el Hedonismo, los dos primeros son adyacentes a los valores motivacionales de la Tradición y la Conformidad; el último es opuesto ${ }^{(12,13)}$. Además, el queso Bola de Ocosingo presentó similaridades con los resultados obtenidos en el queso Chapingo ${ }^{(28)}$, tanto por la presencia de la Benevolencia como un valor motivacional importante en las variables de regresión seleccionadas, como por la manifestación de la misma ruta de influencia (indirecta) de los valores humanos en el consumo de los quesos.

\section{Conclusiones e implicaciones}

Los valores motivacionales importantes que fueron comunes en ambas muestras de consumidores fueron Benevolencia y Seguridad. Los consumidores del queso Crema de Chiapas adicionalmente presentaron como valores motivacionales al Poder y al Universalismo; mientras que los consumidores de queso Bola de Ocosingo presentaron al Hedonismo como un valor suplementario. La influencia de los valores humanos de los consumidores chiapanecos sobre la preferencia del queso Crema de Chipas fue del tipo directa, significa que los consumidores emplearon atributos intangibles (afectivos y simbólicos) además de los tangibles, en la evaluación holística del queso, para su consumo. Para el queso Bola de Ocosingo, la influencia de los valores humanos sobre la preferencia del queso fue indirecta, indicando que para su consumo las personas valoraron únicamente los atributos tangibles, dándole un significado utilitario. Estos resultados implican que, durante el consumo de diferentes variedades de quesos tradicionales mexicanos, la identificación de las rutas directa o indirecta puede aportar información sobre la preferencia de un queso, más allá de sus atributos tangibles. Esto permitiría diferenciar y tipificar a los quesos tradicionales por el tipo de influencia que los valores humanos ejercen en la preferencia de los consumidores. A partir de estos hallazgos, se sugiere colocar signos distintivos en la etiqueta del producto, y planear estrategias 
mercadotécnicas dirigidas a grupos de consumidores diferenciados sociodemográficamente para promover un mayor involucramiento afectivo con el queso. Además de la valorización de los atributos tangibles del queso, la identificación de los significados simbólicos otorgados por los consumidores a los quesos tradicionales mexicanos deberá ser realizada por los investigadores de los quesos tradicionales mexicanos, con una visión integradora e interdisciplinaria.

\section{Literatura citada:}

1. González-Córdova AF, Yescas C, Ortíz-Estrada AM, De la Rosa-Alcaraz MA, Hernández-Mendoza A, Vallejo-Córdoba B. Artisanal Mexican cheeses. J Dairy Sci 2016;99(5):3250-3262.

2. Villegas-de-Gante A, Cervantes-Escoto F, Cesín-Vargas A, Espinoza-Ortega A, Hernández-Montes A, Santos-Moreno A, et al. Atlas de los quesos mexicanos genuinos. 1era ed. México, Biblioteca Básica de Agricultura, Editorial Colegio de Posgraduados; 2014.

3. Guerrero L, Guardia MMD, Xicola J, Verbeke W, Vanhonacker F, Zakowska S, et al. Consumer-driven definition of traditional food products and innovation in traditional foods. A qualitative cross-cultural study. Appetite 2009;52(2):345-354.

4. Kupiec B, Revell B. Specialty and artisanal cheeses today: the product and the consumer. Brit Food J 1998;100(5):236-243.

5. Cesín VA, Aliphat FM, Ramírez VB, Herrera HJG, Martínez CD. Ganadería lechera familiar y producción de queso. Estudio en tres comunidades del municipio de Tetlatlahuca en el estado de Tlaxcala, México. Téc Pecu Mex 2007;45(1):61-76.

6. Espinoza OA, Álvarez MA, Del Valle MC, Chauvet SM. La economía de los sistemas campesinos de producción de leche en el estado de México. Téc Pecu Mex 2005;43(1):39-56.

7. Hirschman E. The creation of product symbolism. Adv Consum Res 1986;13(1):327331.

8. Thompson DMH, Crocker C, Marketo CG. Linking sensory characteristics to emotions: An example using dark chocolate. Food Qual Prefer 2010;21(8):11171125.

9. Firat F, Venkatesh A. Liberatory postmodernism and the re-enchantment of consumption. J Consum Res 1995;22(3):239-267.

10. Allen MW. Human values and product symbolism: do consumers form product preference by comparing the human values symbolized by a product to the human values that they endorse? J Appl Soc Psychol 2002;32(12):2475-2501. 
11. Dreezens EAA, Martijn C, Tenbült P, Kok GJ, de Vries NK. Food and the relation between values and attitudes characteristics. Appetite 2005;45(1):40-46.

12. Schwartz SH. Universals in the content and structure of values: Theoretical advances and empirical tests in 20 countries. Adv Exp Soc Psychol 1992;25:1-65.

13. Schwartz SH. An overview of the Schwartz theory of basic values. Online Read Psychol Cult 2012;2(1):1-20. https://scholarworks.gvsu.ed/cgi/viewcontent.cgi?article=1116\&context=orpc. Accessed Feb 20, 2107.

14. Botonaki A, Mattas K. Revealing the values behind convenience food consumption. Appetite 2010;55(3):629-638.

15. Sashi, Kottala SY, Singh R. A review of sustainability, deterrents, personal values, attitudes and purchase intentions in the organic food supply chain. Pac Sociol Rev 2015;1(3):114-123.

16. Sharma R, Jha M. Values influencing sustainable consumption behavior: Exploring the contextual relationship. J Bus Res 2017;76:77-88.

17. Lee PY, Lusk K, Mirosa M, Oey I. The role of personal values in Chinese consumers' food consumption decisions. A case study of healthy drinks. Appetite 2014;(73):95104.

18. Brunsø K, Scholderer J, Grunert KG. Testing relationships between values and foodrelated lifestyle: results from two European countries. Appetite 2004; (43):195-205.

19. Allen MW. A practical method for uncovering the direct and indirect relationships between human values and consumer purchases. J Consum Mark 2001;12(2):102120.

20. Allen MW, Torres CV. Food symbolism and consumer choice in Brazil. Gonzalez S, Luna D, editors. Duluth, MN, USA: LA Adv Consum Res 2006;(1):180-185.

21. Hayley A, Zinkiewicz L, Hardiman K. Values, attitudes, and frequency of meat consumption. Predicting meat-reduced diet in Australians. Appetite 2015;84:98-106.

22. Lindberg E, Garling T, Montgomery H. Belief-value structures as determinants of consumer behavior: A study of housing preferences and choices. J Consum Policy 1989;12:119-137.

23. Keaveney SM, Hunt KA. Conceptualization and operationalization of retail store image: A case of rival middle-level theories. J Acad Market Sci 1992;20:165-175.

24. Allen MW. The attribute-mediation and product meaning approaches to the influences of human values on consumer choices. Adv Psychol Res 2000;1:31-76. 
25. Schwartz SH. Are there universal aspects in the content and structure of values? J Soc Issues 1994;50:19-45.

26. Chalmer BJ. Understanding statistics. USA. Marcel Dekker Inc.; 1987.

27. Dekking FM, Kraaikamp C, Lopuhaä HP, Meester LE. A modern introduction to probability and statistics: understanding why and how. 1rst ed. New York, USA: Springer; 2005.

28. Hernández-Montes A. Influencia de valores humanos en la aceptación del queso Chapingo y sus significados intangibles de compra. Agr Soc Desarro [en imprenta] 2019. 